\title{
Oxaliplatin, 5-fluorouracil and leucovorin (modified FOLFOX-6) as first-line chemotherapy for advanced gastric cancer patients with poor performance status
}

\author{
HYEONG SU KIM ${ }^{1}$, JUNG HAN KIM ${ }^{1}$, HEE JUN KIM ${ }^{1}$, HYUN JOO JANG ${ }^{1}$, JIN BAE KIM ${ }^{1}$, \\ JI WON KIM ${ }^{2}$, SO YOUNG JUNG ${ }^{2}$, BYUNG CHUN KIM ${ }^{2}$, DAE HYUN YANG ${ }^{2}$, \\ SOAH PARK ${ }^{3}$, KEONG JU KIM ${ }^{3}$, SOON IL LEE $^{4}$ and DAE YOUNG ZANG ${ }^{1}$

\begin{abstract}
Departments of ${ }^{1}$ Internal Medicine, ${ }^{2}$ Surgery, and ${ }^{3}$ Radiation Oncology, Hallym Medical Center, Hallym University College of Medicine, Seoul 150-950; ${ }^{4}$ Department of Hemato-Oncology, Dankook University Hospital, Dankook University College of Medicine, Chunan 330-715, Republic of Korea
\end{abstract}

Received August 1, 2011; Accepted November 17, 2011

DOI: $10.3892 / \mathrm{ol} .2011 .496$

\begin{abstract}
This study was designed to retrospectively evaluate the efficacy and safety of modified FOLFOX-6 regimen in advanced gastric cancer (AGC) patients with poor performance status (PS). From January 2005 to August 2010, 23 AGC patients with poor PS who received mFOLFOX-6 as first-line chemotherapy were reviewed. Patients received $100 \mathrm{mg} / \mathrm{m}^{2}$ oxaliplatin and $100 \mathrm{mg} / \mathrm{m}^{2}$ leucovorin (LV) as a 2 -h intravenous infusion on day 1 , followed by 5 -fluorouracil (5-FU) at $2000 \mathrm{mg} / \mathrm{m}^{2}$ as a 46 -h continuous infusion. A total of 22 patients received more than 3 cycles of chemotherapy and were evaluable for tumor response. Seven patients achieved partial response, giving an overall response rate of $31.8 \%$. The median time to progression and overall survival were 3.5 and 9.2 months, respectively. Grade 3-4 hematological toxicities were noted: neutropenia in four patients $(17.4 \%)$, anemia in two $(8.7 \%)$ and thrombocytopenia in one $(4.3 \%)$. Two patients developed febrile neutropenia and one of these patients succumbed to sepsis. Grade 3-4 gastrointestinal toxicities, such as nausea, vomiting and diarrhea were observed in less than $10 \%$ of patients. Peripheral neuropathy was observed in 9 patients (39.1\%). In conclusion, the mFOLFOX-6 regimen for AGC patients with poor PS was effective with acceptable toxicity. Our results suggest that this regimen may be an effective option for these patients.
\end{abstract}

Correspondence to: Dr Jung Han Kim, Department of Internal Medicine, Kang Nam Sacred-Heart Hospital, Hallym Medical Center, Hallym University College of Medicine 948-1, Daelim-1 Dong, Youngdeungpo-Gu, Seoul 150-950, Republic of Korea E-mail: harricil@hotmail.com

Key words: advanced gastric cancer, modified FOLFOX-6, poor performance status

\section{Introduction}

Gastric cancer is one of the most common types of cancer worldwide in terms of incidence and mortality. In Korea, gastric cancer is the most commonly occurring malignancy, accounting for approximately $15 \%$ of newly diagnosed cancer cases (1). Although its incidence in individuals younger than 50 years has been on the increase, gastric cancer develops more frequently among patients in their seventies and eighties. The only potential curative treatment is surgery; however, a considerable number of patients present with advanced disease at the time of diagnosis. Moreover, more than half of patients treated by complete resection eventually develop recurrent diseases during the course of their condition $(2,3)$. Palliative chemotherapy is known to improve survival in advanced gastric cancer (AGC) when compared with best supportive care (4). However, the prognosis of advanced and recurrent disease remains poor.

Oxaliplatin plus infusional 5-fluorouracil (5-FU) and leucovorin (FOLFOX regimen) is widely used as a first-line treatment in patients with colorectal cancer (5). A few studies have shown the activity and tolerability of the combination of oxaliplatin and 5-FU modulated with leucovorin (FOLFOX-4, modified FOLFOX-4, FOLFOX-6 and modified FOLFOX-6) in patients with AGC (6-11). The reported objective response rate (RR) was 40-50\%, with a median overall survival (OS) of 10 to 12 months. In terms of toxicities, these regimens have been well-tolerated. However, as these studies have been conducted mostly in patients with good performance status (PS), information regarding patients with poor PS is limited.

The aim of this study was to investigate retrospectively the activity and toxicity of a modified FOLFOX-6 (mFOLFOX-6) regimen in AGC patients with poor PS.

\section{Materials and methods}

Patients. Between January 2005 and August 2010, AGC patients who received mFOLFOX-6 as first-line chemotherapy were retrospectively reviewed. To be eligible for the study, patients 
were required to have had histologically confirmed adenocarcinoma or signet ring cell carcinoma of the stomach. The patients were also required to have unresectable or metastatic disease with at least one measurable lesion and a European Clinical Oncology Group (ECOG) PS of 2. In addition, patients had to fulfill the following criteria: age exceeding 18 years; no central nervous system metastasis; no active infection; no history of other malignancies; and sufficient hepatic, renal and bone marrow functions. Patients who had previously been treated with adjuvant chemotherapy were included. This study was approved by the Institutional Review Board (IRB). As this retrospective study would involve no risk of patients, the waiver of patient consent was allowed by the IRB.

Treatment schedule. All 23 patients received $100 \mathrm{mg} / \mathrm{m}^{2}$ oxaliplatin and $100 \mathrm{mg} / \mathrm{m}^{2}$ leucovorin (LV) as a 2-h intravenous infusion on day 1 , followed by $100 \mathrm{mg} / \mathrm{m}^{2} 5-\mathrm{FU} 2000$ as a 46-h continuous infusion. Antiemetic prophylaxis with 5-HT3 antagonists were always administered prior to the oxaliplatin infusion, but corticosteroids were not routinely administered. This regimen was repeated every 14 days to the maximum 12 cycles, unless there was no evidence of disease progression or unacceptable toxicity.

Treatment was delayed for up to 2 weeks until the patient recovered from adverse effects, the absolute granulocyte count exceeded 1,000 $\mu \mathrm{l}$ and/or the platelet count exceeded $100,000 \mu 1$. Dose reductions were made according to the most severe grade of toxicity that had occurred during the previous cycle. The dose of oxaliplatin and 5-FU was reduced by $20 \%$ for hematological or non-hematological toxicity of grade $3-4$. Oxaliplatin was reduced by $20 \%$ in patients with persistent peripheral neuropathy. In case of persistent paresthesias interfering with function, oxaliplatin was omitted until recovery.

Response and toxicity evaluation. Baseline evaluation included physical examination, complete blood counts (CBCs), blood chemistry and radiological examinations. Physical examination, CBC and blood chemistry were performed prior to each cycle. Tumor assessments by CT scans were repeated every 4 cycles. Response was assessed according to the guidelines of the Response Evaluation Criteria in Solid Tumors (RECIST) Committee. Complete response (CR) was defined as the disappearance of all evidence of disease. Partial response (PR) was defined as a reduction of at least $30 \%$ in unidimensional tumor measurements, without any new lesion or progression of any existing lesion. Progressive disease (PD) was defined as an increase of at least $20 \%$ in the sum of the products of all measurable lesions or the appearance of any new lesion, including the reappearance of any lesion that had disappeared. Stable disease (SD) was defined as a tumor response not fulfilling the criteria for CR, PR and PD.

Toxicities, except for peripheral neuropathy, were recorded according to the Common Toxicity Criteria of the National Cancer Institute (NCI-CTC), version 3.0. Peripheral neuropathy was graded according to the following oxaliplatin-specific scale: grade 1, paresthesias or dysesthesias of short duration, but resolving prior to the next cycle: grade 2, paresthesias persisting between two cycles without functional impairment: grade 3, persistent paresthesias interfering with function. For
Table I. Patient characteristics.

No. of patients $(\%)$

Number included

Age, years

Median

61

Range

30-74

Gender

Male

$18(78.3 \%)$

Female

$5(21.7 \%)$

Histology

Adenocarcinoma

$20(87 \%)$

Well-differentiated

$7(30.4 \%)$

Moderately differentiated

$3(13 \%)$

Poorly differentiated

$10(43.4 \%)$

Signet ring cell carcinoma

$3(13 \%)$

Disease status

Recurrent

$8(34.9 \%)$

Metastatic

$13(56.5 \%)$

Locally advanced

$2(8.7 \%)$

Sites of metastases

Liver

$7(30.4 \%)$

Lymph node

$8(34.9 \%)$

Lung

$5(21.7 \%)$

Peritoneum

$10(43.3 \%)$

Ovary

$2(8.7 \%)$

Bone

$2(8.7 \%)$

Prior adjuvant chemotherapy

None

$18(78.3 \%)$

Yes

$5(21.7 \%)$

toxicity analysis, the worst data for each patient in all cycles of chemotherapy were used.

Statistical analysis. Response and toxicity data were analyzed using simple descriptive statistics. Time to progression (TTP) was determined from the first day of chemotherapy to disease progression or death. OS was calculated from the first day of treatment to the date of last follow-up or death. Survival curves were established by using the Kaplan-Meier method.

\section{Results}

Patient characteristics. A total of 23 patients were included in this study. Their baseline characteristics are shown in Table I. The patients comprised 18 male (78.3\%) and 5 female $(21.7 \%)$ patients with a median age of 61 years (range, 30-74). Of the 23 patients, 20 patients $(87 \%)$ had adenocarcinoma and the remaining 3 had signet ring carcinoma. The most common metastatic site was the peritoneum (43.3\%), followed by the abdominal lymph node (34.9\%), liver (30.4\%) and lung $(21.7 \%)$. Of the 23 patients, 8 (34.9\%) had recurrent disease following curative resection and 15 had metastatic or locally 
Table II. Toxicity profile by grade $(n=23)$.

\begin{tabular}{|c|c|c|}
\hline Toxicity & Grade 1-2 No. (\%) & Grade 3-4 No. (\%) \\
\hline \multicolumn{3}{|l|}{ Hematological toxicities } \\
\hline Neutropenia & $8(34.8 \%)$ & $4(17.4 \%)$ \\
\hline Febrile neutropenia & - & $2^{\mathrm{a}}(8.7 \%)$ \\
\hline Anemia & $10(43.5 \%)$ & $2(8.7 \%)$ \\
\hline Thrombocytopenia & $5(21.7 \%)$ & $1(4.3 \%)$ \\
\hline \multicolumn{3}{|c|}{ Non-hematological toxicities } \\
\hline Nausea & $10(43.3 \%)$ & $2(8.7 \%)$ \\
\hline Vomiting & $7(30.4 \%)$ & $2(8.7 \%)$ \\
\hline Hand-foot syndrome & $2(8.7 \%)$ & 0 \\
\hline Diarrhea & $3(13.0 \%)$ & $1(4.3 \%)$ \\
\hline Mucositis & $4(17.4 \%)$ & 0 \\
\hline Peripheral neuropathy & $8(34.8 \%)$ & $1(4.3 \%)$ \\
\hline Allergic reaction & $2(8.7 \%)$ & 0 \\
\hline Fever & $2(8.7 \%)$ & - \\
\hline
\end{tabular}

${ }^{\mathrm{a} O n e}$ patient succumbed to sepsis.

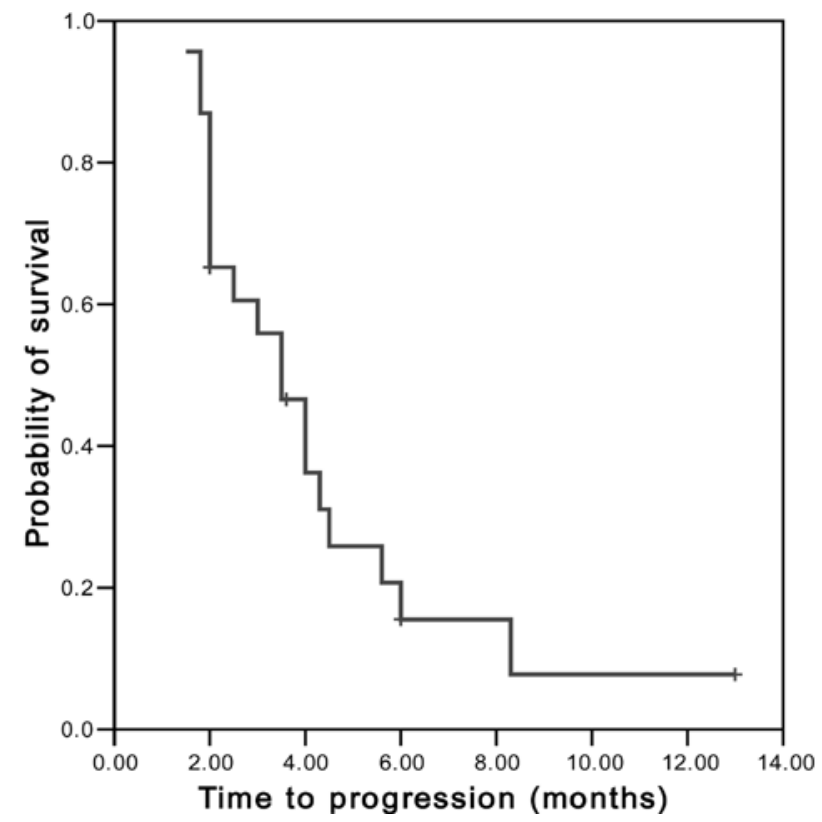

Figure 1. Time to progression.

advanced disease. Out of 8 patients with recurrent disease, 5 had received adjuvant chemotherapy.

Tumor response and survival. A total of 148 cycles were delivered, with a median number of 7 cycles per patient (range, 2-12). One patient was not evaluable for tumor response due to a lung abscess, which developed following 2 cycles of chemotherapy. Four patients (17.4\%) received only 3 cycles of chemotherapy due to clinical impairment in 3 patients and hypersensitivity reaction in 1 patient. Of the 23 patients, 22 received $>3$ cycles of chemotherapy and were assessable for tumor response evaluation. Seven patients achieved PR, yielding an overall RR

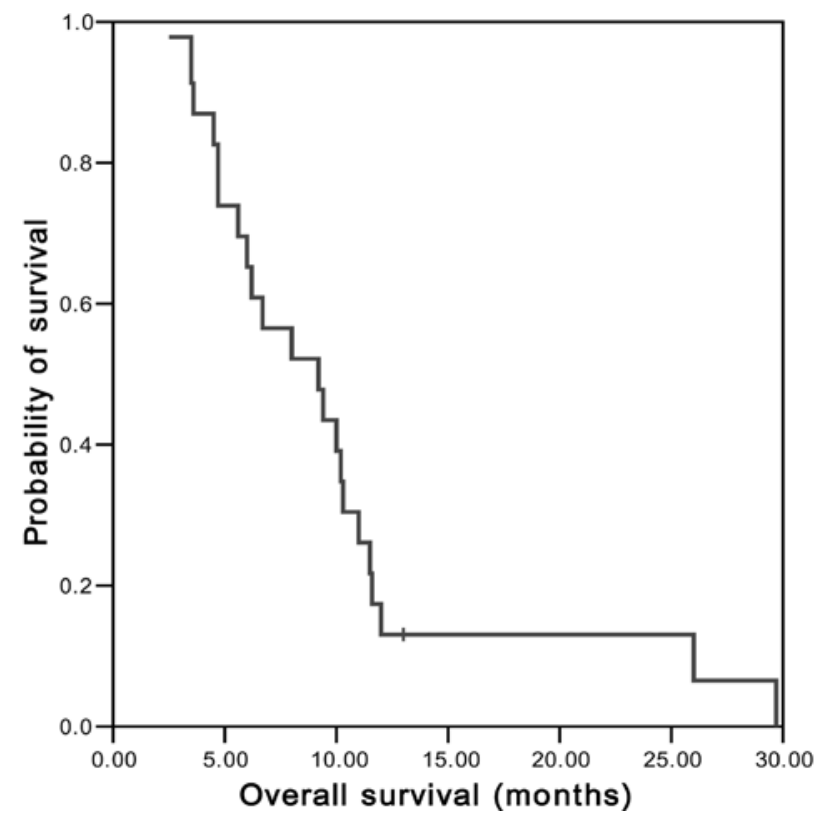

Figure 2. Overall survival.

of $31.8 \%$. Six patients $(27.3 \%)$ exhibited SD and the remaining $8(36.4 \%)$ exhibited PD.

With a median follow-up of 9.2 months, the median TTP was 3.5 months (range, 1.5-13.0), Fig. 1. The median OS was 9.2 months (range, 2.5-29), with a 1-year survival rate of $13 \%$ (Fig. 2).

Toxicities. All 23 patients were evaluable for toxicity. The toxicity profile is shown in Table II. Four patients $(17.4 \%)$ delayed the following cycle of chemotherapy due to myelosuppression. Five patients $(21.7 \%)$ required dose reduction due to grade 3-4 toxicities. Grade 3-4 hematological toxicities were as 
follows: neutropenia in 4 patients $(17.4 \%)$, anemia in $2(8.7 \%)$ and thrombocytopenia in 1 patient (4.3\%). Two patients developed febrile neutropenia, and 1 patient succumbed to sepsis.

Non-hematological toxicities were generally mild. Grade 1-2 nausea, vomiting and diarrhea were observed in $10(43.4 \%)$, $7(30.4 \%)$ and $3(13 \%)$ patients, respectively. Grade 3-4 gastrointestinal toxicities were as follows: nausea in 2 patients, vomiting in 2 and diarrhea in 1 patient. Grade 1-2 peripheral neuropathy was observed in 8 patients $(34.8 \%)$, while grade 3 peripheral neuropathy occurred in 1 patient. Peripheral neuropathy led to a dose reduction in 2 patients and treatment discontinuation in 1 patient. Two patients developed allergic reaction during the administration of oxaliplatin in the third and eleventh cycle, respectively. Symptoms and signs included skin rash, pruritis, chest discomfort, tachycardia and hypotension, which were resolved by discontinuation of oxaliplatin and administration of steroids and antihistamines. No more chemotherapy was administered in these 2 patients.

\section{Discussion}

During the past decade, new chemotherapeutic agents, such as docetaxel, paclitaxel, capecitabine, S-1, irinotecan and oxaliplatin, have been developed and tested extensively in AGC. Although AGC is considered to be chemo-sensitive with an overall RR ranging between 30 and $60 \%$, its prognosis has remained poor, with a median OS of approximately 10 months. Gastric cancer develops more frequently among elderly patients (1). Additionally, a considerable number of patients with metastatic or recurrent gastric cancer have poor PS in association with previous gastrectomy or co-morbidity. Therefore, development of optimal regimens for those patients is crucial.

Since the combination of oxaliplatin with LV and 5-FU (FOLFOX-6) has demonstrated good activity and an acceptable safety profile for patients with AGC (6), we treated AGC patients with poor PS using the mFOLFOX-6 regimen (biweekly with $100 \mathrm{mg} / \mathrm{m}^{2}$ oxaliplatin, $100 \mathrm{mg} / \mathrm{m}^{2} \mathrm{LV}$ and $2000 \mathrm{mg} / \mathrm{m}^{2}$ 5-FU). We used an infusional 5-FU regimen due to the lower hematological toxicity, compared to bolus 5 -FU $(12,13)$. However, since severe gastrointestinal toxicities such as mucositis and diarrhea were expected with the infusional 5-FU, we administered $100 \mathrm{mg} / \mathrm{m}^{2} \mathrm{LV}$ instead of $400 \mathrm{mg} / \mathrm{m}^{2}$ in the FOLFOX-6 regimen.

A variety of FOLFOX regimens have demonstrated good activity and tolerability for patients with AGC (6-11). However, as the enrolled patients had mostly good PS, information regarding AGC patients with poor PS is limited. In this study, we reviewed the efficacy and safety of the mFOLFOX-6 regimen for AGC patients with poor PS. The overall RR was $31.8 \%$, with a median TTP of 3.5 months and a median OS of 9.2 months. As expected in patients with poor PS, these results are inferior to those in other studies with various FOLFOX regimens (6-11). However, the clinical outcomes in our study are comparable to those reported in a phase II study of mFOLFOX-4 for gastric cancer patients with malignant ascites (14). In the study by Oh et al, approximately $50 \%$ of the enrolled patients (22/48) had poor PS (ECOG 2) (14). Of the 36 patients with measureable lesions, 12 patients exhibited PR, yielding an overall RR of 33.3\%. The median TTP and OS observed in all 48 patients were 3.5 and 8.4 months, respectively. Therefore, considering that all patients in the current study had poor PS, our results are encouraging.

In terms of toxicity, the mFOLFOX-6 regimen used in the current study was acceptable. Four patients delayed their next cycle of therapy owing to myelosuppression and 5 patients required dose reduction due to grade 3-4 toxicities or persistent neuropathy. Although there was one treatment-related death, hematological toxicities were manageable. The incidence of grade 3-4 myelotoxicities in the present study was similar to those reported in other regimens that employed the same drugs (6-12). The most common non-hematological toxicities were nausea (52.1\%) and vomiting (39.1\%). However, grade 3-4 non-hematological toxicities (nausea, vomiting, diarrhea and peripheral neuropathy) were observed in less than $10 \%$ of patients. Peripheral neuropathy occurred more frequently as compared to other studies $(8,14)$, which appears to be associated with a high dose of oxaliplatin $\left(100 \mathrm{mg} / \mathrm{m}^{2}\right)$ in the present study.

In conclusion, the mFOLFOX-6 regimen used in our study was both active and acceptable for AGC patients with poor PS. Our results suggest that this regimen may be an effective option for those patients.

\section{References}

1. Jung KW, Park S, Kong, HJ, et al: Cancer statistics in Korea: incidence, mortality, survival, and prevalence in 2008. Cancer Res Treat 43: 1-11, 2011.

2. Greenlee RT, Murray T, Bolden S and Wingo PA: Cancer statistics. CA Cancer J Clin 50: 7-33, 2000.

3. Gunderson LL and Sosin H: Adenocarcinoma of the stomach: areas of failure in a reoperation series (second or symptomatic look) - clinicopathologic correlation and implications for adjuvant therapy. Int J Radiat Oncol Biol Phys 8: 1-11, 1982.

4. Catalano V, Labianca R, Beretta GD, et al: Gastric cancer. Crit Rev Oncol Hematol 54: 209-241, 2005.

5. de Gramont A, Figer A, Seymour M, et al: Leucovorin and fluorouracil with or without oxaliplatin as first-line treatment in advanced colorectal cancer. J Clin Oncol 18: 2938-2947, 2000.

6. Louvet C, Andre T, Tigaud JM, et al: Phase II study of oxaliplatin, fluorouracil, and folinic acid in locally advanced or metastatic gastric cancer patients. J Clin Oncol 20: 4543-4548, 2002.

7. Al-Batran SE, Atmaca A, Hegewisch-Becker S, et al: Phase II trial of biweekly infusional fluorouracil and folinic acid, and oxaliplatin in patients with advanced gastric cancer. J Clin Oncol 22: 658-663, 2004.

8. De Vita F, Orditura M, Matano E, et al: A phase II study of biweekly oxaliplatin infusional 5-fluorouracil and folinic acid (FOLFOX-4) as first-line treatment of advanced gastric cancer patients. Br J Cancer 92: 1644-1649, 2005.

9. Luo HY, Xu RH, Zhang L, et al: A pilot study of oxaliplatin, fluorouracil and folinic acid (FOLFOX-6) as first-line chemothetapy in advanced or recurrent gastric cancer. Chemotherapy 54: 228-235, 2008.

10. Liu ZF, Guo QS, Zhang XQ, et al: Biweekly oxaliplatin in combination with continuous infusional 5-fluorouracil and leucovorin (modified FOLFOX-4 regimen) as first-line chemotherapy for elderly patients with advanced gastric cancer. Am J Clin Oncol 31: 259-263, 2008.

11. Lee HH, Hur H, Kim SH, Park AR, Kim W and Jeon HM: Outcomes of modified FOLFOX-6 as first line treatment in patients with advanced gastric cancer in a single institution; retrospective analysis. Cancer Res Treat 42: 18-23, 2010.

12. Diaz-Rubio E: New chemotherapeutic advances in pancreatic, colorectal, and gastric cancers. Oncologist 9: 282-294, 2004.

13. Ibranhim A, Hirschfeld S, Cohen MH, et al: FDA drug approval summaries: oxaliplatin. Oncologist 9: 8-12, 2004.

14. Oh SY, Kwon HC, Lee S, et al: A phase II study of oxaliplatin with low-dose leucovorin and bolus and continuous 5-fluorouracil (modified FOLFOX-4) for gastric cancer patients with malignant ascites. Jpn J Clin Oncol 37: 930-935, 2007. 